vol. $33-n^{\circ} 2$ et $3 \mid 2017$

Dire la violence des frontières. Mises en mots de la migration vers l'Europe

\title{
Cuche Denys, La notion de culture dans les sciences
} sociales. Cinquième édition

\section{Charlotte Vampo}

\section{(2) OpenEdition}

\section{Journals}

Édition électronique

URL : https://journals.openedition.org/remi/8623

DOI : $10.4000 /$ remi.8623

ISSN : $1777-5418$

Éditeur

Université de Poitiers

Édition imprimée

Date de publication : 1 septembre 2017

Pagination : 342-344

ISBN : 979-10-90426-59-7

ISSN : 0765-0752

Référence électronique

Charlotte Vampo, "Cuche Denys, La notion de culture dans les sciences sociales. Cinquième édition », Revue européenne des migrations internationales [En ligne], vol. 33 - n² 2 et 3 | 2017, mis en ligne le 01 septembre 2017, consulté le 14 avril 2022. URL : http://journals.openedition.org/remi/8623 ; DOI : https://doi.org/10.4000/remi.8623 


\section{Notes de lecture}

\section{Flory, Céline}

De l'esclavage à la liberté forcée. Histoire des travailleurs africains engagés dans la Caraïbe française au XIXe siècle. - Paris : Khartala : Société des africanistes, 2015. - $455 \mathrm{p}$. ISBN : 978-2-8111-1339-1

Ce livre, consacré à I'histoire des travailleurs africains engagés dans la Caraïbe française au XIXe siècle, est issu d'une thèse de doctorat en histoire couronnée par deux prix en 2011 et 2012. Alors que l'esclavage a été très largement étudié, la catégorie des travailleurs engagés sous contrat l'est beaucoup moins. En réalité, celle-ci recouvre deux sous-populations distinctes. Sur les 18520 personnes introduites avant et après l'abolition de l'esclavage en 1848 en Martinique, en Guadeloupe et en Guyane dans la Caraïbe, la plus grande partie (93 \%) était des individus libres qui avaient signé un contrat dit d'engagement, $7 \%$ des hommes, des femmes et des enfants rachetés par des armateurs français, alors qu'ils étaient captifs ou esclaves en Afrique.

Si Céline Flory parle de liberté forcée, c'est parce que le parallèle avec l'esclavage s'impose. Sans être captif esclave, ni esclave, ils n'étaient nullement maîtres de leur corps, ni de leur mouvement, en particulier par rapport à la possibilité de retourner en Afrique à la fin de leur contrat. Les engagés, en dépit des apparences, avaient le plus souvent signé ce contrat sans vraiment en connaître les conditions. Ils n'étaient donc pas " maîtres de leur volonté ". Quant à ceux qui avaient été rachetés par des engagistes, ceux pour qui ils devaient travailler, ils furent utilisés comme une main-d'œuvre quasi servile, les employeurs s'efforçant d'éviter l'accusation d'esclavage, dans le contexte abolitionniste de la première moitié du XIXe siècle.
L'intérêt de l'ouvrage est de présenter de manière très approfondie et documentée pratiquement toutes les dimensions du problème ou précisément tous les acteurs impliqués. Au XVlle et XVIlle siècle, au moment de l'esclavage le pouvoir des planteurs était quasiment illimité, la royauté, beaucoup trop lointaine, était incapable d'exercer un contre-pouvoir réel. Au XIXe siècle, la situation internationale est différente. Le gouvernement français doit constamment être attentif à la pression exercée par l'Angleterre et aux accords internationaux relatifs à l'esclavage signés par la France. Ainsi un droit de visite pouvait être exercé par la Royal Navy sur les navires français transportant les engagés.

La première partie du livre, centrée sur les tensions entre la France et l'Angleterre et illustrée par les échanges de correspondance entre les ministres des deux pays, équilibre remarquablement I'histoire diplomatique et des relations internationales et celle des rivalités économiques autour de l'enjeu crucial de l'approvisionnement en main-d'œuvre dans les colonies. Par exemple, l'Angleterre qui bénéficiait de l'immense réservoir de main-d'œuvre en Inde ne laissa quasiment aucun accès aux recruteurs français. Un chapitre intitulé " Une immigration africaine transatlantique obsolète " met en lumière les conséquences de la montée en puissance de l'empire colonial français en Afrique. Les besoins en main-d'œuvre de l'économie de plantation dans les Antilles deviennent secondaires comme l'exprime bien Faidherbe en 1857 : " L'intention du gouvernement français ne peut être de sacrifier ses colonies pleines d'avenir de la côte occidentale d'Afrique, à ses colonies des Antilles, qui ont dit depuis longtemps leur dernier mot, en fait de développe- 
ment et toujours causé tant de difficultés à la métropole pour ces malheureuses questions d'esclavage et des haines des races. $»$

La deuxième partie analyse les conditions concrètes de la filière migratoire et le rôle des quatre entrepreneurs privés, en montrant le contrôle exercé par le gouvernement français, qui institue des " délégués du gouvernement pour les migrations ", aussi appelés agentschirurgiens, car ils appartenaient au corps des chirurgiens de la Marine, pour vérifier les engagements au départ des côtes africaines et qui embarquaient sur les bateaux jusqu'à l'arrivée aux Antilles, d'où souvent des conflits avec les capitaines. L'auteur distingue à juste titre la question du consentement dans le cas des Africains libres (1854-1857) et dans celui des Africains captifs (1857-1862). L'analyse très détaillée des conditions concrètes de la signature du contrat d'engagement sur ces deux périodes montre bien le piège qui se refermait sur les hommes et les femmes libres, surtout des Krous. Les Africains captifs furent rachetés plutôt au Gabon et au Congo, mais l'offre disponible était constituée d'individus souvent malades, affaiblis, chétifs, au point que les recruteurs français n'arrivaient pas à tenir les engagements conclus contractuellement avec le gouvernement, d'autant qu'ils étaient en concurrence avec les négriers transatlantiques. La réalité de la condition des rachetés, la procédure de rachat sont telles que la distance par rapport à l'esclavage est bien ténue. Le principe même d'un consentement, qui figure pourtant dans le décret du 27 mars 1852 qui organisait le rachat, ne fut nullement respecté. Les conditions abominables de la traversée, sur des bateaux dont l'auteur décrit très précisément l'organisation de l'espace, aboutirent à une mortalité élevée qui rappelle encore fortement esclavage : emplacement, entassement, modalités de conservation de l'eau, latrines favorisaient les épidémies de dysenterie, tandis que I'habillement dérisoire et l'alimentation insuffisante aggravaient encore les risques de mortalité. Et comme pour les esclaves, la discipline était très dure, entraînant parfois des rébellions.

La troisième partie porte sur la vie dans les colonies. L'étude des conditions de travail est centrée sur le travail forcé, qui permettait de maintenir l'organisation économique alors que l'esclavage avait été aboli, avec des salaires dérisoires qui maintenaient de fait les engagés dans la misère, compte tenu de tous les prélèvements effectués et de la quasi-impossibilité de pouvoir racheter le retour à la fin du contrat d'engagement. La vie dans les colonies se caractérise aussi par des formes de résistance : protestations auprès des autorités, auxquelles il était rarement donné suite, révoltes et violences physiques, fuite et " marronnage ", suicides. Le dernier chapitre porte sur les relations avec la société créole. Les esclaves africains se mêlaient parfois, mais pas toujours aux Créoles et aux travailleurs indiens, et ceci davantage en Guyane qu'en Guadeloupe et en Martinique.

En résumé, ce livre, remarquablement documenté et enrichi de nombreux tableaux, graphiques, cartes et d'une abondante bibliographie, est une contribution majeure à la connaissance de cette période de transition entre l'esclavage et la reconnaissance à la fin du siècle du statut de citoyens à toute cette population d'immigrés présents depuis plusieurs décennies dans les colonies. On ne peut que saluer le tour de force qui consiste à proposer, on l'a dit, une vision globale de cette page de I'histoire coloniale, tout en apportant sur chacun de ses aspects des analyses précises qui emportent la conviction.

Yves Charbit

Professeur émérite de démographie CEPED/Université Paris Descartes 
Pieret, Denis

Les frontières de la mondialisation. Gestion des flux migratoires en régime néolibéral. Liège, Presses Universitaires de Liège, 2016. $-328 \mathrm{p}$. ISBN : 978-2-87562-095-8

Dans l'article qu'elle a publié à l'occasion des dix ans de la revue Mobilities, Sheller (2016) appelait la communauté scientifique à utiliser une approche foucaldienne afin de mettre à jour les dispositifs qui jouent sur les inégalités d'accès à la mobilité. Denis Pieret, docteur en philosophie de l'université de Liège, répond avec brio à cet appel dans le livre adapté de sa thèse de doctorat, Les frontières de la mondialisation. Gestion des flux migratoires en contexte néolibéral.

La question qui traverse ce travail est celle de la rationalité qui sous-tend la gestion contemporaine des flux migratoires et l'auteur la pose à travers la thématique plus générale des effets du passage du libéralisme au néolibéralisme sur les populations migrantes. Pour y répondre, il se fixe plusieurs objectifs. D'abord, dans une approche foucaldienne du pouvoir, montrer comment fonctionne la norme (dans ses dimensions souveraine, disciplinaire et biopolitique) dans le champ des politiques migratoires. Ensuite, comprendre les contradictions apparentes qui structurent les politiques migratoires contemporaines sous l'angle de leur participation à une rationalité cohérente, celle du néolibéralisme qui exerce toujours le pouvoir à la fois de manière répressive et incitative. Enfin, il entend montrer que la gestion contemporaine des flux migratoires multiplie les espaces et les formes d'action de la frontière, et ce faisant, agit en produisant des mobilités différenciées selon un nouveau principe structurant : non plus intérieur vs extérieur, mais mobile vs fixe.

Pour remplir ses objectifs, Denis Pieret articule données empiriques (en s'appuyant sur l'étude de documents officiels, institutionnels, juridiques, etc.) et cadre philosophique, lui-même adossé à deux courants théoriques distincts : celui, issu des travaux de Foucault, qui analyse le néolibéralisme comme une gouvernementalité, et celui qui porte sur l'étude de la mondialisation et met en évidence la multiplication des centres et des périphéries (notamment les travaux de Balibar, Harvey, Wallerstein).

La première partie du livre retrace dans une perspective généalogique les grands principes du droit des étrangers (illustré par le cas de la Belgique), de la libre circulation et des politiques d'asile et d'immigration en Europe depuis la signature du Traité de Rome jusqu'au programme de Stockholm.

La deuxième partie du livre offre un rappel bienvenu de la conceptualisation foucaldienne des différentes technologies de pouvoir, et sert de prélude à l'analyse des différents instruments de gestion des mouvements migratoires. Pieret commence par un passage en revue des instruments répressifs du pouvoir, ceux qui marquent des frontières : les camps, les zones extraterritoriales, les centres de rétention, les murs, les programmes qui s'appuient sur les nouvelles technologies pour ériger des " smart borders ". II note cependant que la fonction de ces outils anciens du pouvoir a tendance à changer : les camps par exemple ne servent plus vraiment à normer le comportement des migrants, mais plutôt à réguler leurs mouvements.

La troisième partie du livre s'intéresse à la nouveauté des discours portant sur le " migration management " en tant qu'il se présente comme un nouveau champ de connaissance qui agence conceptions théoriques, outils de surveillance, dispositifs administratifs, aspects juridiques et techniques de la frontière. Pieret isole et interroge la rationalité managériale de ce nouveau savoir spécialisé. 
La quatrième et dernière partie du livre porte sur la manière dont les frontières agissent comme instrument de différenciation visant à maintenir en mouvement ou à contraindre à l'immobilité les populations en fonction des exigences d'un capitalisme global.

Le travail de Pieret tend finalement à montrer comment le nouveau régime de régulation des migrations vise à faire des migrants, selon les préceptes de la gouvernementalité néolibérale, des entrepreneurs de soi, des hommes parfaitement adaptés à cette nouvelle logique de flux qui agit non pas en mettant tout et tous indifféremment en mouvement, mais qui fluidifie en " stasiant " différentiellement, en aménageant les conditions qui favoriseront seulement la mobilité des plus aptes. Ce faisant, la hiérarchisation de la population passe désormais par la dichotomie mobile-fixe, " bons " migrants qu'il faut encourager et laisser circuler, et " mauvais " migrants qu'il faut " stasier " ou maintenir dans la périphérie.

En conclusions, le livre de Denis Pieret nous offre une perspective originale sur les enjeux qui sous-tendent les régulations contemporaines des flux migratoires, en même temps qu'une opérationnalisation très réussie de la pensée du dernier Foucault. En sortant d'une philosophie " auto-centrée ", Pieret réussit son pari de nous offrir une réflexion philosophique poussée sur un objet empirique à l'actualité brûlante.

Nous retenons en particulier son articulation claire et ambitieuse des différents concepts qui composent le champ contemporain des politiques migratoires et leur mise en perspective à la lumière d'une théorisation de la rationalité frontalière. Nous regrettons parfois le manque d'approfondissement de certains concepts ou idées, mais cela est à porter au compte d'une pensée riche qui aborde un nombre conséquent d'éléments propres à ce nouveau management des migrations.
Finalement, nous rejoignons Balibar qui signe la postface du livre et qui écrit : " la maîtrise du sujet, l'érudition pluridisciplinaire, la clarté d'exposition, la construction d'une argumentation à la fois nuancée et progressive, la fermeté de l'orientation théorique proposée font du livre de Denis Pieret un travail de très grande qualité ". Nous recommandons la lecture de ce livre à tous ceux qui désirent comprendre en profondeur cette nouvelle gestion des migrations en contexte néolibéral, ses apparentes contradictions et sa rationalité propre.

\section{Référence bibliographique}

Sheller Mimi (2016) Uneven Mobility Futures: A Foucauldian Approach, Mobilities, 11 (1), pp. 15-31.

Cécile Giraud

Doctorante et assistante

Centre d'études du développement/ Université Catholique de Louvain

Whongo Ahanda, Antoine

Comment s'informent et communiquent

les Camerounais de l'étranger. - Paris,

L'Harmattan, 2014. - 218 p.

ISBN : 978-2-343-03994-7

Dans son ouvrage court et dense intitulé Comment s'informent et communiquent les Camerounais de l'étranger? Antoine Whongo Ahanda, journaliste de formation alors conseiller culturel à I'ambassade du Cameroun à Paris depuis une quinzaine d'années, nous propose une approche de la migration camerounaise saisie au prisme des outils disciplinaires des sciences de l'information et de la communication. II prend particulièrement appui sur les effets des nouvelles technologies de l'information et de la communication (NTIC) ayant permis au courant migratoire camerounais de contourner les traditionnels obstacles à sa visibilité, ou tout du moins à son audibilité, afin d'opérer son incursion dans l'arène des affaires publiques de son pays d'origine, 
plus particulièrement l'arène politique que l'auteur choisit de scruter prioritairement. Pour ce faire, il place ses travaux sous l'angle d'une confrontation conceptuelle entre communication et transnationalisme, confrontation qu'il considère à même de mieux rendre compte des constructions diasporiques atypiques, voire marginales, en cours au sein de ce courant migratoire, particulièrement en île-de-France où la majeure partie de son travail a été conduite.

Cet ouvrage est structuré en cinq études suivant une logique propre aux ouvrages collectifs hétérogènes, chaque section ou chapitre disposant de sa propre bibliographie. II démarre par une introduction générale permettant à l'auteur d'exposer dans un premier temps, les justifications de cette production scientifique dont la plus prégnante serait la mondialisation. Cette dernière est considérée comme point d'ancrage de la diversité des modes de communication transnationale en œuvre dans les nouvelles constructions diasporiques multiformes. C'est le cas des démarches subversives des composantes migratoires camerounaises considérées marginales qu'il met en résonance avec l'image fortement écornée du pays d'origine à l'étranger. Dans un second temps de cette introduction générale, il présente la méthodologie adoptée. Celle-ci croise dépouillement de bases documentaires diverses, entretiens semi-structurés auprès de Camerounais de la région francilienne et observations participantes en tant qu'acteur de premier plan, tout en convoquant la dimension pluridisciplinaire de la mouvance des "cultural studies" dans laquelle l'auteur ancre la filiation de ses travaux et tente ainsi d'expliquer le caractère juxtaposé des thématiques évoquées dans les différentes parties intitulées " étude ".

À cet effet, la première étude s'attache à formuler un " transnationalisme camerounais " que l'auteur estime enchâssé entre un désir d'intégration de ces migrants en pays d'accueil et une revendication de citoyenneté considérée " à distance " par rapport au pays d'origine, à laquelle il attribue la notion de " citoyenneté externe ॥. II s'appuie sur la trajectoire géo-historique du Cameroun, particulièrement sur sa dimension politique fortement oppressive tant en période coloniale que postcoloniale, pour expliquer la mise en place d'un courant migratoire camerounais aussi appelé " mouvements diasporiques camerounais " fortement contestataire. II procède dans un premier temps à la restitution de I'histoire politique de cette diaspora en nous éclairant sur la mise en place d'une opposition camerounaise portée en migration par I'Union Nationale des Étudiants Kamerunais (UNEK). Cette opposition est dès le départ soumise à une stratégie croisée de séduction-répression parfois féroce sous le règne du premier président Ahmadou Ahidjo (1960-1982), aboutissant à ce que l'auteur appelle " maquis intellectuel ". Un assouplissement des relations survient avec l'avènement de la politique du "Renouveau " du second président Paul Biya (en place depuis 1982). À la fin des années 1980, cet assouplissement est accéléré par les divers changements globaux ayant imposé l'adoption des lois renforçant les libertés publiques au Cameroun en concomitance avec l'émergence des NTIC. Ensuite l'auteur nous propose un panorama de la migration camerounaise en établissant une catégorisation traversée par la double facette classique des migrations sur le plan juridique (régulières et irrégulières). Cette catégorisation repose sur des effectifs estimatifs dans lesquels on retrouve trois composantes majeures que sont les migrations pour études et la problématique des retours, les migrations de travail et la problématique des transferts de fonds, et les migrations familiales avec la problématique des compositions matrimoniales transnationales. Le panorama proposé est ensuite renforcé par une esquisse de la hiérarchisation des espaces d'accueil de ces migrants dont l'auteur 
essaye d'éclairer les différents modes d'organisation tant associatifs que politiques faits de clivages et convergences en vue de l'exercice de la citoyenneté, voire de la "formation d'une identité de citoyen ".

Cette première étude s'achève par une esquisse de l'enjeu politique de la diaspora camerounaise que constituent la participation politique et ses processus électoraux nourrissant un transnationalisme politique. Celui-ci développe dans ses diverses revendications politiques redoutées par le pouvoir en pays d'origine qui continue $d^{\prime} y$ apporter des réponses inconstantes, bien souvent défensives, voire teintées de " nationalisme gouvernemental ". Ce qui, selon l'auteur, fait cohabiter en pays d'accueil face au processus d'intégration, deux principales figures de cette migration, à savoir les " assimilés " par adoption de la nationalité et les "non-assimilés " à fort sentiment d'appartenance les conduisant à réclamer à distance l'exercice de la citoyenneté.

La deuxième étude s'inscrit dans le prolongement de la précédente. Sur la base des critères d'honnêteté, compétence et « nationalisme " structurant une enquête qualitative effectuée auprès de migrants camerounais vivant en région parisienne, elle nous livre les opinions politiques défavorables portées sur l'ensemble des hommes politiques camerounais considérés véreux et corrompus, qu'ils soient du parti au pouvoir ou de l'opposition. Ce qui lui permet de dresser l'état des lieux de la perception tant du parti au pouvoir usant de son statut de privilégié pour adopter une attitude équivoque face au respect du jeu démocratique, que de l'opposition conduite par des dirigeants jugés peu sérieux, ne présentant pas de différence notoire avec ceux du parti au pouvoir et par conséquent aussi considérés inaptes à gouverner le Cameroun. L'auteur peut alors dégager une catégorisation des préférences partisanes de Camerounais de l'étranger structurée en trois groupes avec un léger avantage pour celui que l'auteur appelle I'" opposition ", suivi du parti au pouvoir et enfin, un groupe sans opinion. Cette catégorisation est affinée en fonction des classes d'âges, du niveau scolaire et de la durée du séjour en France, au même titre que la structuration du choix politique de ces migrants que l'auteur place sous emprise de quatre facteurs que sont l'information, l'intérêt personnel, le profil du candidat et l'idéologie défendue. Ces deux derniers facteurs semblent primer dans le choix des Camerounais de l'étranger avec une nette préférence pour le facteur idéologique.

L'information qui semble ainsi conditionner très faiblement le choix des migrants camerounais interrogés constitue l'élément majeur de la troisième étude consacrée aux méthodes de communication d'une frange marginale de cette migration. La notion d'" exopolitie " est alors convoquée pour désigner les actions de communication s'appuyant sur Internet considéré comme nouveau territoire de lutte d'opposants activistes en exil. À ce titre, l'auteur nous livre quelques figures majeures de ces exilés regroupés au sein d'un mouvement fédératif d'associations à objet politique et à caractère progressiste, alternatif, voire transgressif, appelé le CODE (collectif des organisations démocratiques et patriotiques de la diaspora) soutenu par certains sites internet dédiés au Cameroun. II rend compte des ressorts et fondements de l'action de ce collectif consistant en une opposition frontale et systématique au régime de Yaoundé soutenue à la fois par une surexploitation des opportunités numériques offertes par Internet dans ses principaux espaces d'ancrages que sont les pays occidentaux, et une mobilisation de quelques anciens exilés de la période de l'indépendance, considérés dépositaires des fonds symboliques de la contestation du pouvoir camerounais avec son renversement pour ultime objectif. Leurs modes d'action prennent corps autant dans des manifestations publiques récurrentes fortement redoutées par les autorités diplomatiques 
camerounaises, que dans le déploiement d'une diplomatie non gouvernementale faite de sollicitations récurrentes d'organisations institutionnelles des territoires d'ancrage par des contacts directs sur demandes d'audiences, ainsi qu'à travers des lettres ouvertes et communiqués de presse relayés grâce aux rencontres et forums. Ces derniers évènements sont aussi autant d'occasions de prôner l'action judiciaire internationale, en même temps que sont scandés les récits historiques en hommage aux martyrs de l'indépendance. Ils donnent aussi lieu à l'organisation récurrente de votes symboliques en faveur de l'alternance au Cameroun, diffusés sur divers supports et canaux tels que portails, sites internet et blogs. Cet activisme médiatique repose par ailleurs sur une surexploitation politique de la notion de diaspora dont l'ampleur aboutit selon l'auteur à la décrédibilisation et la dénaturation de la notion de "diaspora " finalement au Cameroun, associée à celle $d^{\prime}$ " opposition radicale ". En somme, il s'agit pour une frange contestataire et radicale des migrants camerounais d'utiliser Internet pour vaincre les obstacles de la distance traditionnellement imposée par les pouvoirs publics dans l'accès à l'arène politique du pays d'origine où elle compte exposer une réalité cachée développée dans la quatrième étude au titre bien évocateur d'agenda setting du Cameroun et sa diaspora médiatique.

Cette quatrième étude s'attarde sur le principe d'« agenda setting ", ou système orienté de production et de diffusion d'une image générale donnée, en l'espèce ici celle du Cameroun en Occident, dont les points d'ancrage sont les différents indicateurs de son sous-développement. Ces indicateurs influencent les sources d'information débouchant sur une image contrastée du Cameroun dans les médias internationaux bien souvent constituée de stéréotypes médiatiques. On se situerait ainsi à la source de la perception généralement négative de ce pays dans le monde, prolongée par l'attitude complaisante, voire complice de sa diaspora, plus particulièrement les méthodes des journalistes en exil et autres personnalités médiatiques de cette communauté. Ce qui conduit I'auteur à conclure cette quatrième étude par des recommandations aux pouvoirs publics de Yaoundé quant à la stratégie à adopter pour mieux gérer l'image du pays confrontée aux nuisances du règne d'Internet, marquant ainsi autrement la transition avec la dernière étude de son ouvrage.

La cinquième étude est consacrée à la réception de la télévision camerounaise par les Camerounais de l'étranger dans un contexte de concurrence de plus en plus accrue. Leur choix alors porté sur le " bouquet africain " traduit selon l'auteur une quête d' " identité culturelle " concernant dans une moindre mesure leurs enfants. Cette dernière étude qui clos l'ouvrage laisse quelques regrets autant du fait de l'absence d'une conclusion générale en résonance à l'introduction générale, qu'en ce qui concerne le modèle de construction hétérogène du document exposant l'auteur à de nombreuses redondances alourdissant le suivi du raisonnement envisagé. À cet effet, les nombreuses répétitions de titres dans les multiples listes bibliographiques proposées, et le profilage contradictoire de la communauté camerounaise apparaissant tant dans la première que dans la dernière étude sont autant d'éléments susceptibles d'égarer le lecteur. L'auteur aurait gagné à consolider au préalable les données disponibles afin d'échapper au périlleux exercice consistant à mobiliser des données différentes pour tenter de construire un raisonnement scientifique cohérent sur le même objet de recherche, en l'occurrence ici la diaspora camerounaise. On peut aussi regretter une posture parfois subjective et partisane de l'auteur, inhérente aux écueils méthodologiques de conduite de recherche en immersion avec une trop forte implication du chercheur. Le biais scientifique se ressent à partir du titre de couverture qui ambitionne de traiter des Camerounais 
de l'extérieur, alors que le texte se confine pour une grande part, au traitement des relations du pouvoir central de Yaoundé avec une frange marginale de ce courant migratoire auquel une généralisation trop hâtive des résultats court le risque d'aboutir à des conclusions fortement discutables, voire erronées.

Néanmoins, ces remarques majeures n'occultent nullement l'intérêt d'une telle approche de la question de la diaspora camerounaise dont l'enjeu identitaire bien adressé reste vissé dans son différend majeur avec les autorités du pays d'origine, à savoir l'exercice des droits civiques en migration bridé par un code de la nationalité interdisant et réprimant la double nationalité. En tout état de cause, cet ouvrage reste une source d'informations non négligeable, avec de très intéressants points d'ancrage pour de sérieuses pistes de recherches originales possibles, et par conséquent, hautement recommandable.

Pierre Kamdem Professeur de géographie MIGRINTER/Université de Poitiers

Halkias, Daphne Adendorff, Christian

Governance in Immigrant Family business. Enterprise, Ethnicity and Family Dynamics. Surrey : Gower. - 2014, 212 p.

ISBN : 978-1-4094-4557-9

Governance in Immigrant Family business est un ouvrage publié aux éditions Gower dans la collection « applied research ". Cette précision annonce la tonalité de l'ouvrage, à la fois effort de cadrage théorique, et essai de recommandations en vue de soutenir et améliorer le fonctionnement des entreprises de migrants. Les auteurs de cet ouvrage sont par ailleurs un chercheur de l'Université de Bergame et un entrepreneur qui mène une activité de consultant sur les questions de gouvernance en entreprise. Ce format, plutôt inhabituel dans le paysage français, fait l'intérêt du livre. Le lecteur ne trouvera pas là un exposé universitaire appuyé sur un projet de recherche, mais plutôt un manuel à direction tant des étudiants que des entrepreneurs ou des pouvoirs publics.

Le livre comporte deux sections. La première rassemble une série de quatre chapitres qui définissent un corpus conceptuel à même de cerner toutes les dimensions de cet objet hybride. II s'agit là de la section qui intéressera le plus le lecteur universitaire. La seconde section (chapitres 5 à 9) passe en revue les défis qui peuvent affecter cette forme d'entreprises, défis relatifs aux dynamiques familiales, entrepreneuriales et culturelles de ce type d'activité. L'originalité de l'approche réside dans la prise en compte de toutes les clés de lecture de l'entreprise familiale de migrants. Contrairement à la plupart des travaux portant sur ce phénomène, ce dernier n'est pas pris comme un pendant du processus migratoire, mais comme un cas particulier de la forme la plus répandue d'activité lucrative au monde, à savoir l'entreprenariat familial. Le second chapitre est entièrement dédié à cet entreprenariat familial, extrêmement répandu (entre 65 et $90 \%$ des entreprises dans le monde). Les auteurs rappellent qu'il n'existe pas de définition universellement admise de I'entreprise familiale tant les formes qu'elle peut prendre sont diverses, allant de la direction effective par une même famille à la simple prise de participation à une affaire dont la gestion est déléguée à d'autres personnes. Les relations qui soudent les membres d'une famille constituent la principale force de ces entreprises. Les relations de confiance, la fierté collective, l'ancrage de ces relations dans la durée sont autant de ferments qui stabilisent le commerce. Mais ces relations constituent également sa principale faiblesse (un tiers seulement sont transmises à la génération suivante). Cette fragilité n'a pas d'autre 
source que le dualisme constitutif de cette forme d'entreprenariat, à la fois affaire à but lucratif et activité fédérative d'une famille. La précédence des obligations familiales peut parfois entrer en contradiction avec les impératifs de performance : privilégier la stabilité d'une activité au détriment de la prise de risque, opter pour un partenaire familial au détriment d'un autre plus compétent. L'existence d'une entreprise peut également être suspendue à la qualité des relations entre ses membres. Un conflit, un divorce ou un décès sont autant d'événements susceptibles de la déstabiliser. Les questions de succession apparaissent comme le talon d'Achille principal. La passation à un nouveau membre ou leur retrait de la gestion effective sont souvent objets de conflits entre partenaires.

Les chapitres 3 et 4 portent plus spécifiquement sur le commerce familial des étrangers. Ils reviennent sur les traits qui le caractérisent, à savoir une propension plus importante à ouvrir ses activités sur l'international grâce à l'expérience migratoire des entrepreneurs ; l'importance du lien culturel qui unit ses partenaires, mais qui peut être un obstacle à l'insertion sur le marché intérieur. L'entreprenariat immigré est souvent lié à la difficulté pour les migrants de s'insérer sur le marché du travail du pays d'accueil. On le sait, cet entreprenariat augmente en période de crise, tandis que les étrangers sont les premiers touchés par le chômage. Toutefois les auteurs soulignent que de plus en plus de migrants utilisent l'entreprenariat non comme une voie de sortie, mais comme un objectif en soi. Les auteurs parlent de migration d'opportunité. Cette tendance, en hausse depuis deux décennies, est liée à la hausse du niveau d'éducation chez les migrants. Cette pratique s'observe en particulier parmi les acteurs qui migrent pour acquérir une formation professionnelle ou académique et qui restent dans le pays d'accueil pour ouvrir une affaire. Internet a démultiplié les possibilités de ce type d'ac- tivité en permettant à moindres frais une projection vers l'international. La littérature distingue les acteurs en fonction de leur ancrage international : entrepreneurs diasporiques qui s'appuient sur des réseaux de la diaspora d'appartenance ; entrepreneurs globaux dont les activités n'ont pas de pays d'ancrage spécifique ; entrepreneurs transnationaux qui focalisent leurs échanges avec le pays d'origine. Avec cette nouvelle forme de commerce, l'expérience migratoire devient constitutive de l'orientation et de la nature des activités.

En définitive, le principal intérêt de cet ouvrage réside dans le panorama conceptuel qu'il propose. Les spécialistes à la recherche d'une enquête et de questionnements originaux n'y trouveront pas leur compte. Des études de cas apparaissent à la fin de chaque chapitre. Elles illustrent avec un bonheur inégal les discussions conceptuelles présentes dans le corps du texte, mais elles ne constituent aucunement une base empirique des réflexions. II faut davantage l'aborder comme une sorte de manuel. Les chapitres passent en revue les principales approches : I'entreprenariat familial et ses paradigmes, l'entreprenariat ethnique et ses concepts (enclave, théorie du " middle-man "), mais aussi l'interférence de la culture dans la gouvernance d'entreprise, etc. Ce livre invite également les spécialistes du commerce ethnique à aborder cet objet comme ancré dans une réalité qui n'est pas spécifique au monde des migrations : le commerce ethnique est aussi un commerce familial comme les autres. II invite donc à se pencher sur une littérature de la sociologie et du management des entreprises qui est trop souvent absente des bibliographies sur le sujet.

Thomas Lacroix

Chargé de recherche, géographe MIGRINTER/Université de Poitiers 
Pérez Siller, Javier (dir.)

Lassus, Jean-Marie (dir.)

Les Français au Mexique, XVIIle-XXIe siècle, vol. 1 et 2. - Paris : L'Harmattan. - 2015,

409 p. (vol. 1) ; 491 p. (vol. 2)

ISBN : 978-2-343-05608-1 (vol. 1) ; 978-2-343-

05607-4 (vol. 2)

Ces deux volumes, qui totalisent près de 900 pages, témoignent de l'ambition d'un projet de longue haleine qui a mobilisé les travaux d'une équipe franco-mexicaine sur plus d'une décennie. II fait suite à plusieurs volumes marquant les étapes de ce projet (1998, 2004, 2010 et 2014) également dirigés par Javier Pérez Siller dans une série intitulée México-Francia : memoria de una sensibilidad común, siglos XIX-XX. Ce projet d'envergure mise sur le long terme et l'accumulation d'enquêtes pour faire émerger une "sensibilité commune " produite par les relations franco-mexicaines et autour de ce que l'on connaît comme l'" afrancesamiento " de la période du Porfiriat au Mexique (sous la présidence de la République de Porfirio Diaz, 1876-1910). Envisageant des phénomènes bien au-delà et en deçà de cette période, l'ouvrage propose non seulement une somme de connaissances sur les relations franco-mexicaines, les migrations françaises au Mexique (peu connues) et I'influence française au Mexique, mais surtout une approche historiographique qui articule histoire culturelle et histoire des sensibilités.

Cette approche est nécessairement subtile, car de fait, les flux migratoires en eux-mêmes sont faibles, tant à l'échelle du Mexique (voir le chapitre de Délia Salazar sur la période de plus forte influence, c'està-dire sous le Porfiriat) que de la migration française atlantique. Pourtant, le Mexique fait bonne figure américaine dans ces différentes expériences migratoires, celles des " communautés " et autres utopies et phalanstères du début du XIXe siècle ou simples projets de colonisation rurale (sur les rives du Rio Coatzacoalcos, ou celles du Rio Nautla étudiées par Patrick Lafarge). Quelques rares filières se mettent en place, principalement celle des barcelonnettes (du nom de la ville des Alpes du sud d'où s'organise cette migration de commerçants au milieu du XIXe siècle, et qui donne lieu à d'importants réseaux marchands et industriels au Mexique), tandis que dans certaines petites régions françaises qui envoient des migrants (Thônes, vallée de I'Ubaye, Hasparren dans le Pays basque) le Mexique fait office d'Amérique.

Si les flux sont peu significatifs, on est tout de même saisi par ces microhistoires, comme par le cas de la colonie de Jicaltepec (Jean-François Campario), par les sources mobilisées qui restituent la fragilité de " l'homme blanc sous les tropiques " et le poids de l'aventure pionnière, encore plus à travers les échecs relatés et la forte mortalité qui caractérise ces populations, immigrées au Mexique ou retournées en France.

D'où la gageure du projet d'articuler ces flux à la notion d' " afrancesamiento ", autrement dit l'influence d'une présence et culture française et les interactions qui forment une "sensibilité commune " (Javier Pérez Siller). Un aspect mieux connu de la présence française vient de sa position dans l'économie atlantique. À ce titre, l'analyse des compagnies maritimes du Havre et de Saint-Nazaire (Veronique Basille Reyes) est très éloquente. Mais là encore, cette influence ne se traduit pas dans l'importance des flux commerciaux ni des flux de migration. Ces derniers favorisent certaines niches professionnelles qui ont été déterminantes pour la présence culturelle française (ce qui n'est pas particulier au Mexique) : boulangers et instituteurs (Maria Isabel Monroy Castillo, Estela Munguía). II s'avère finalement que cette présence française au Mexique, bien que discrète, permet à un voyageur suisse en 1855 de parcourir une bonne partie du pays en passant d'une maison française à I'autre (Guy Rozat). 
Certes, la filière des barcelonnettes qui reste la principale expérience de la migration française au Mexique, bien plus que l'épisode scabreux de l'empire de Maximilien (1864-1867), a conduit à la présence de Français parmi les élites commerçantes et industrielles. En tant que tels, ces migrants participent à une culture nationale d'autant que la plupart se marient sur place et s'apparentent alors aux grandes familles mexicaines, plus acculturés qu'acculturant.

Comment alors mesurer et voir ces sensibilités françaises au Mexique prendre forme ? C'est l'objet du second volume qui rejoint une historiographie de l'histoire des savoirs et techniques et du transfert technologique et culturel dans la mondialisation. On apprend beaucoup sur la culture française et la vision française du monde : la diffusion du restaurant (Raquel Barceló Quintal) ou de la géographie par exemple (Ferderico Fernández Christlieb), sont révélées par leur réception mexicaine. Pour le restaurant en particulier, on y voit la formation d'un modèle dans les sociabilités urbaines qui permet de dégager une réflexion générale sur les métropoles latino-américaines au XIXe siècle.

Malgré l'expérience, ou plutôt à cause, de l'empire avorté de Maximilien, du destin de ses soldats (Solène Garotin), on constate une relative timidité des interactions avec la France. Celle des élites est plutôt celle d'une internationalisation qui se produit à Paris (Victor Macías) et éventuellement dans des salons " relais " à Mexico, et celle des classes populaires est trop peu nombreuse pour avoir des retentissements sensibles. À ce titre, I'expérience de Léon Martin, petit employé des barcelonnettes, est remarquable (Leticia Gamboa). À travers l'interprétation de sa correspondance avec ses parents en France, on perçoit une expérience traumatisante, celle de l'enfermement du subalterne, broyé dans la machine du grand magasin qui n'a rien à envier à l'ouvrier captif des ateliers parisiens, new-yorkais ou de São Paulo. Dans ce kaléidoscope d'expériences et d'études, on peut regretter une mise en perspective dans les sociétés mexicaines et françaises, qui permettraient de rendre la singularité de tel ou tel témoignage et son interprétation pour l'analyse des sensibilités franco-mexicaines qui est l'objet du livre et du projet. En tout cas, celui-ci impressionne par son mérite - moins par sa facture - à constituer cette somme d'expériences, de collectes, de regards, de données qui intéresseront en soi les historiens de la France et du Mexique et qui donnent matière à réflexion, hors des projecteurs de la migration européenne vers l'Argentine, les États-Unis ou le Brésil, sur la formation d'une culture atlantique originale au cours du XIXe siècle, où, malgré d'évidentes inégalités économiques et géopolitiques entre la France et le Mexique, les rôles nationaux, raciaux et ethniques ne sont pas encore tout à fait distribués par des rapports de force de type postcolonial.

Aurélia Michel

Maître de conférences en histoire CESSMA/Université Paris-Diderot

Bonnet, Éric (dir.)

Soulages, François (dir.)

Frontières et artistes. Espace public, mobilité et (post)colonialisme en Méditerranée. -

Paris : L'Harmattan. - 2014, 175 p.

ISBN : 978-2-343-04914-4

Fruit d'une rencontre internationale, ce livre collectif explore les relations que les artistes originaires des pays méditerranéens tissent avec les frontières, les influences que les migrations, volontaires ou non, exercent sur les œuvres et sur l'imaginaire. Les auteurs se demandent ce qu'est " une pratique géoartistique de la mobilité "(p. 7), ce qu'induisent les convulsions du colonialisme et du postcolonialisme, ce que les contacts culturels entraînent en matière d'hybridation artis- 
tique. Les créateurs veulent se situer " à partir d'une philosophie du devenir et non d'une philosophie de l'être, une philosophie du flux et non une philosophie de la permanence " (p. 16). L'ouvrage invoque le patronage d'Ulysse, vu comme le héros paradigmatique de la mobilité en Méditerranée, artiste de sa propre vie dont il a fait une performance. D'Ulysse on passe à Du Bellay qui célébra le « beau voyage " qu'accomplit le héros d'Homère ; la leçon en est que la mobilité constitue une voie d'accès privilégiée à la formation et ensuite à la création.

L'artiste est influencé par la globalisation; il se détache de ses racines locales et transporte des signes qu'il mêle à ce qu'il rassemble au cours de son errance. II abandonne parfois la peinture pour réaliser des installations dans lesquelles s'interpénètrent les images et les influences qu'il a rencontrées. Ainsi le créateur s'affranchit des fixités et offre des œuvres mouvantes. La subjectivité n'en est pas absente car l'artiste puise aussi dans son expérience personnelle formée au fil de ses déplacements. Certains proposent des photos de lieux connus ou de foules anonymes, photos enrichies de commentaires manuscrits, de documents divers faisant connaître les sentiments ou les activités du photographe. D'autres, dans le sillage de Matisse ou de Klee, travaillent au métissage des codes orientaux et occidentaux. Ces formes nouvelles, qualifiées de " post-orientalistes ", engendrent une culture plurielle. On peut aboutir à une sorte de narration constituée de matériaux recyclés ou à une cartographie voulant rendre compte d'un rêve, d'une critique du pouvoir, de la restitution d'une réalité sociale. L'ordinateur devient parfois un outil de médiation artistique.

Les messages que veulent transmettre les auteurs ne sont pas toujours limpides car " les frontières interdisent parfois I'universalité au profit de I'hybride contemporain qui unit, paradoxalement parfois, uniformité et communautarisme " (p. 15). Les artistes et esthètes qui ont contribué à la rédaction du livre utilisent souvent une langue obscure reflétant les complexités des concepts qu'ils manient. Autre difficulté : ils décrivent généralement des œuvres qu'ils ne reproduisent pas, faute de moyens matériels. Mais, si l'on dépasse les gongorismes de l'expression et si l'on imagine ce que l'on ne voit pas, on perçoit l'intérêt de la démarche qui essaie de caractériser la création artistique à l'époque de la mondialisation.

Ralph Schor

Professeur émérite d'histoire Université de Nice-Sophia-Antipolis

\section{Cuche, Denys}

La notion de culture dans les sciences sociales. Cinquième édition. - Paris : La Découverte, 2016. - $172 \mathrm{p}$. ISBN : 978-2-7071-9059-8

On ne présente plus l'ouvrage de Denys Cuche sur la notion de culture devenu un " classique dans son genre " comme le mentionnait déjà Gajardo (2010) à l'occasion de la publication de la quatrième édition du livre en 2010. Incontournable dans la formation des étudiants en sciences sociales et utile aux chercheurs les plus expérimentés, il est remis au goût du jour dans une cinquième édition de 172 pages (la première datant de 1996). On notera tout de suite l'ajout d'un chapitre VII (p. 116) sur l'extension de la notion de culture à de nouveaux champs d'application tel que le développement, ainsi que le transfert des parties sur les cultures des immigrés et le multiculturalisme présentes dans le chapitre sur les enjeux sociaux de la notion de culture de la quatrième édition dans un chapitre autonome (chapitre IX, p. 142) intitulé « Migrations internationales et transformations culturelles ".

L'introduction de cette édition de même que les cinq premiers chapitres sont similaires à ceux de la précédente. Le propos introductif visant à montrer 
que l'on n'a jamais autant parlé de culture qu'aujourd'hui notamment de culture des médias, de la jeunesse et des immigrés, prend appui sur une citation de Augé (1988). Le mot « aujourd'hui " gagne ainsi à être relativisé. À partir de cette citation, l'auteur fait état de l'utilisation incontestée du terme (p. 5) compris au sens étendu comme " modes de vie et de pensée ". Toutefois, il rappelle que la notion n'a pas toujours été admise depuis son apparition au XVIlle siècle et qu'une étude de l'évolution historique du concept scientifique est nécessaire (p. 6). II procède alors à la revue des différents sens donnés à la culture entre les chapitres I et $V$ en montrant que les luttes de définitions sont en fait des luttes sociales. De cette manière, il fait le pont entre les débats théoriques et leurs implications concrètes ; exercice fondamental pour les étudiants qui ne saisiraient pas tous les enjeux de ces débats d'apparence déconnectés des réalités sociales.

Le chapitre VI présente l'intérêt de traiter du lien entre culture et identité mais notre attention doit surtout être portée sur le nouveau chapitre VII du livre dans lequel sont présentés les nouveaux champs d'application de la notion de culture. L'auteur en détermine deux principaux : le développement et la démographie. Pour le premier, un rapport tel que celui du Programme des Nations unies pour le développement (PNUD) de 2004 cité dans l'ouvrage et intitulé " La liberté culturelle dans un monde diversifié " souligne que le développement est aussi une question culturelle. Existe-t-il des obstacles culturels au développement ? C'est en tout cas une question bien connue des anthropologues (p. 119) et posée indirectement par les agents du développement lorsqu'ils parlent de " résistance culturelle " au développement. Denys Cuche reprend les travaux de Olivier de Sardan (1995) pour rappeler qu'il n'y a pas de culture inapte au développement. II met en garde contre la vision fixiste de la culture qui fait disparaître l'acteur derrière une " culture impersonnelle " (p. 120). On retiendra de ce point sur la culture et le développement que la culture est un élément stratégique, dynamique et en renouvellement. Elle fait aussi son entrée dans le champ de la démographie nous dit l'auteur en citant les travaux de Charbit et Petit (2012) qui suggèrent une démographie compréhensive dite " démographie anthropologique ». Dans cette approche, il s'agit d'intégrer le facteur culturel dans les schémas explicatifs des comportements démographiques, de rendre compte de la rationalité des acteurs et de désamorcer finalement la critique d'une science quantitative détachée des faits sociaux. C'est un appel à repenser les disciplines anthropologiques, démographiques et l'interdisciplinarité auquel l'auteur semble adhérer tout en émettant une réserve partagée par certains anthropologues face au risque d'une définition de la culture restreinte à des indicateurs listés grossièrement dans des questionnaires (p. 123).

La notion de "culture d'entreprise " fait ensuite l'objet du chapitre VIII. Son intégration dans l'édition de 2010 constituait d'ailleurs son intérêt principal. On constate dans la nouvelle édition toutefois qu'elle n'est pas incluse dans le chapitre sur les nouveaux champs sociaux de diffusion de la notion de culture ; considérons donc que le lien entre entreprise et culture est acquis. Un encadré sur le management interculturel a cependant été ajouté. Les exemples du contremaître animant une équipe d'ouvriers immigrés et d'un(e) chef(fe)s de projet en informatique qui gère du personnel en Inde sont utilisés pour montrer le défi commun des dirigeant(e) $s$ à gérer les différences de cultures dans le cadre de leur travail. Depuis les années 1980 en France, la thématique entreprise/ culture était exploitée en effet par les sociologues sous l'angle des confrontations culturelles entre les groupes sociaux qui composent l'entreprise en fonction de leurs rôles (p. 134). Denys Cuche reprenant les travaux de Chevrier (2013) nous indique les éléments qui caractérisent ce nouveau management interculturel absent de la dernière édition. 
Le choix d'élaborer un chapitre final (chapitre IX) sur les migrations internationales et les transformations culturelles est intéressant à observer au regard de l'actualité française et européenne. La polémique médiatisée au cours de l'été 2016 autour du burkini (le maillot de bain qui recouvre le corps de femmes musulmanes de manière complète) sur les plages de France a soulevé des questions sur le vivre ensemble, sur le respect des cultures et du corps des femmes, de ladite " culture d'origine " et sur l'intégration dans un espace national multiculturel. Plus que jamais, l'ouvrage de Denys Cuche nous rappelle d'être prudents avec ces termes. Une société multiculturelle ne $s^{\prime}$ inscrit pas forcément dans un modèle multiculturaliste et la notion de culture d'origine présente des limites que nous ne pouvons qu'inviter à lire. Un(e) immigré(e) n'est pas non plus totalement doté(e) d'une culture importée d'un autre endroit qui le/la rend imperméable à la société dans laquelle il/elle vit. Ces précisions sur la notion de culture (et les mots qui en découlent comme celui "d'interculturel ") faites au prisme de la migration ont toutes leur pertinence dans un contexte national et européen de peur exacerbée de l'autre.

Quant à la conclusion de l'ouvrage, cette dernière reste inchangée. Elle se base sur un paradoxe qui est celui de la réticence d'un côté de certains chercheurs à utiliser le concept de culture si ce n'est sous la définition stricte du mot, c'est-àdire « de productions intellectuelles et artistiques " (p. 157) du fait de la complexité à s'entendre sur son sens et d'un autre côté de la diffusion toujours plus grande de la notion de culture dans les milieux sociaux. Face à cette réticence, l'auteur nous alerte sur le danger de l'abandon du concept au nom de l'accumulation des connaissances et il défend l'idée, sans doute prin- cipale du livre, de l'utilité du concept pour les sciences sociales. Ce dernier est utile pour reconsidérer la question du relativisme culturel et pour repenser la notion d'ethnocentrisme définis comme étant des " principes méthodologiques " après que leurs limites aient été dressées (p. 161). Cette approche originale, en plus de tous les enseignements que l'on peut tirer de l'ouvrage, mérite tout particulièrement I'attention des étudiants qui sont amenés très souvent à penser l'ethnocentrisme comme un biais de la recherche à éviter et dont on ne peut rien extraire alors que c'est en comparant le mode de vie de l'autre à sa propre expérience sociale que l'on peut espérer une bonne compréhension des phénomènes culturels.

\section{Références bibliographiques}

Augé Marc (1988) L'autre proche, in Martine Segalen Dir., L'Autre et le semblable. Regards sur l'ethnologie des sociétés contemporaines, Paris, Presses du CNRS, pp. 19-34.

Charbit Yves et Petit Véronique (2012) Culture, rationalité et démographie compréhensive, in Erwan Diantell Dir., La Culture et les sciences de l'homme. Un dialogue avec Marshall Sahlins, Paris, Archives Karéline, pp. 187-200.

Chevrier Sylvie (2013) Le management interculturel, Paris, Presses Universitaires de France, 127 p.

Gajardo Anahy (2010) Denys Cuche, La notion de culture dans les sciences sociales, Revue Européenne des Migrations Internationales, 26 (3), pp. 218-221.

Olivier De Sardan Jean-Pierre (1995) Anthropologie et développement, Paris, Karthala, 224 p.

Charlotte Vampo Doctorante en anthropologie CEPED/Université Paris Descartes 